\title{
COMPLETENESS OF METRIZABLE PRE-IMAGES OF VAN DOUWEN-COMPLETE SPACES
}

\author{
KÔICHI TSUDA
}

(Communicated by Franklin D. Tall)

\begin{abstract}
We shall show the recurrence of complete metrizability of irreducible closed pre-images of van Douwen-complete spaces. As its corollary we shall show that every van Douwen-complete space is $G_{\delta}$ in any Lašnev space.
\end{abstract}

\section{INTRODUCTION AND PRELIMINARIES}

All spaces in this paper are assumed to be Tychonoff, and all maps are assumed to be continuous. A space $Y$ is a Lašnev (respectively, van Douwencomplete) space provided that there exists a closed onto map $f: X \rightarrow Y$, where $X$ is a (respectively, completely) metrizable space. By the following fact, it is equivalent to also require that the map $f$ be irreducible, i.e., $f(A) \neq Y$ for any proper closed subset $A$ of $X$.

Fact 0 [5]. For every closed mapping $f: X \rightarrow Y$ from a metric space $X$ onto $Y$, there exists a closed set $A$ of $X$ such that $f \mid A: A \rightarrow Y$ is a closed irreducible onto map.

It is known that van Douwen-complete spaces have some properties in common with Čech-complete spaces. For example, the Baire category theorem is valid for both of them [9, 13] (see also Remark 1(b)). On the other hand, a van Douwen-complete space cannot be Čech-complete unless it is metrizable (see also Example 2 below). Hence, Čech-completeness is not preserved by a closed irreducible mapping. However, our main theorem shows that it remembers completeness in the sense that arbitrary closed irreducible metrizable pre-images must be complete.

Theorem 1. Let $f: M \rightarrow Y$ be a closed irreducible onto map from a complete (in particular, locally compact) metric space $M$, and suppose that $h: X \rightarrow Y$ is an arbitrary closed irreducible map from a metric space $X$ onto $Y$. Then $X$ is completely (respectively, locally compact) metrizable.

The following three facts are fundamental tools for a proof of the above theorem.

Received by the editors July 29, 1993 and, in revised form, January 10, 1994.

1991 Mathematics Subject Classification. Primary 54E50, 54E40, 54C10, 54F45.

Key words and phrases. Complete metric space, closed map, irreducible map, Čech-completeness, universal space. 
Fact 1 [3, Lemma 4.4.16]. Let $f: X \rightarrow Y$ be a closed map of a metric space onto a space $Y$. Then, Bdry $f^{\leftarrow}(y)$ is compact if $y$ has a countable neighborhood base.

Fact 2 [6]. Let $f: X \rightarrow Y$ be a closed map from a paracompact space $X$ onto a space $Y$. Then, for any compact $K \subset Y$, there exists a compact $A \subset X$ such that $f(A)=K$.

Fact 3 [3, Theorems 3.7.21, 3.7.24 and 3.9.10]. If $X$ and $Y$ are Tychonoff spaces and there exists a perfect mapping from $X$ onto $Y$, then $X$ is Čechcomplete (respectively, locally compact) if and only if $Y$ is Čech-complete (respectively, locally compact).

\section{Proofs of OUR ReSULTS}

Lemma 1. Let $f: M \rightarrow Y$ be a closed irreducible onto map from a complete metric space $M$, and suppose that $h: X \rightarrow Y$ is a closed irreducible onto map from a metric space $X$. Then, there exists a dense $G_{\delta}$-subset $Y_{0}$ of $Y$ such that

(a) both $f \mid M_{0}: M_{0} \rightarrow Y_{0}$ and $h \mid X_{0}: X_{0} \rightarrow Y_{0}$ are homeomorphisms, where $M_{0}=f^{\leftarrow}\left(Y_{0}\right)$ and $X_{0}=h^{\leftarrow}\left(Y_{0}\right)$;

(b) $y \in Y_{0}$, whenever $\operatorname{Int}\left(h^{\leftarrow}(y)\right) \neq \varnothing$.

Proof. For each $n \geq 1$ let $G_{n}=\left\{y \in Y\right.$ : diam $\left.\left(f^{\leftarrow}(y)\right)<1 / n\right\}$, and let $H_{n}=\left\{y \in Y: \operatorname{diam}\left(h^{\leftarrow}(y)\right)<1 / n\right\}$. Then, at first we shall show that $H_{n}$ is open in $Y$. Since $h$ is a closed map, for a given $y_{0} \in H_{n}$, there exists its open neighborhood $V$ in $Y$ satisfying that

$$
h^{\leftarrow}(V) \subset B_{\varepsilon}\left(h^{\leftarrow}\left(y_{0}\right)\right),
$$

where $\varepsilon=1 / 2 n-d / 2, d=\operatorname{diam}\left(h^{\leftarrow}\left(y_{0}\right)\right)$, and $B_{\varepsilon}(A)$ is the $\varepsilon$-neighborhood of the set $A$. Then, $V \subset H_{n}$ by the direct computation of the diameters of the fibers of each $y \in V$.

Next we shall show that $f^{\leftarrow}\left(H_{n}\right)$ is dense in $M$. Indeed, let $U$ be an arbitrary non-empty open set of $M$. Then, there exists a fiber $f^{\leftarrow}(y) \subset U$, since $f$ is irreducible. Hence, there exists an open neighborhood $V$ of $y$ in $Y$ such that $f^{\leftarrow}(V) \subset U$. For the open set $h^{\leftarrow}(V)$, take a non-empty open subset $U^{\prime}$ in $X$ such that $U^{\prime} \subset h^{\leftarrow}(V)$ and diam $U^{\prime}<1 / n$. Then, by a parallel argument applying for the open set $U^{\prime}$ and the map $h$, we have a non-empty open set $V^{\prime}$ of $Y$ such that $h^{\leftarrow}\left(V^{\prime}\right) \subset U^{\prime}$. Note that $V^{\prime} \subset H_{n}$. Therefore,

$$
U \cap f^{\leftarrow}\left(H_{n}\right) \supset f^{\leftarrow}\left(V^{\prime}\right) \neq \varnothing .
$$

By a parallel argument each $f^{\leftarrow}\left(G_{n}\right)$ is also open dense in $M$ (see also [10]). Let

$$
M_{0}=\left(\cap_{n} f^{\leftarrow}\left(G_{n}\right)\right) \cap\left(\cap_{n} f^{\leftarrow}\left(H_{n}\right)\right) \text { and } Y_{0}=f\left(M_{0}\right) \text {. }
$$

Then by the Baire category theorem, $M_{0}$ is a dense subset of $M$, since $M$ is complete. Therefore (a) holds by the definition of $G_{n}$ and $H_{n}$. The set $Y_{0}$ is a dense $G_{\delta}$ subset of $Y$, since $f$ is continuous and the following equality holds:

$$
Y_{0}=\left(\cap_{n} G_{n}\right) \cap\left(\cap_{n} H_{n}\right) .
$$

Suppose that $\operatorname{Int}\left(h^{\leftarrow}(y)\right) \neq \varnothing$. Then, it is a one-point set by the irreducibility of $h$. Hence, $h^{-}(y)$ is an isolated point of $X$ and $y$ is also an isolated point in $Y$ since $h$ is a closed map. This completes (b), since $Y_{0}$ is dense in $Y$. 
Proof of Theorem 1. Let $X_{0}, M_{0}$, and $Y_{0}$ be the dense $G_{\delta}$-subsets, satisfying Lemma 1. Put $Z_{0}=\left\{(t, x) \in M \times X: f(t)=h(x) \in Y_{0}\right\}$ and $Z=\{(t, x) \in$ $\left.M \times X:(t, x) \in \operatorname{cl}\left(Z_{0}\right)\right\}$. Then note that $Z \subset\{(t, x) \in M \times X: f(t)=h(x)\}$. Let $\alpha: Z \rightarrow M$ and $\beta: Z \rightarrow X$ be the restrictions of natural projections (i.e., $\alpha(t, x)=t$ and $\beta(t, x)=x)$. Then, by Fact 3 it suffices to show that both of $\alpha$ and $\beta$ are perfect onto maps.

(i) $\alpha$ is onto. Suppose that $t \in M \backslash M_{0}$. Since $M_{0}$ is dense in $M$, there exists a sequence $\left\{t_{n}\right\} \subset M_{0}$ converging to $t$. Put $y_{n}=f\left(t_{n}\right)$ and $y=f(t)$. Then by Fact 2 there exists a compact set $K \subset X$ such that

(1) $h(K)=\{y\} \cup\left\{y_{n}: n \in \omega\right\}$.

Since $t \in M \backslash M_{0}$, we can assume that $\left\{y_{n}\right\}$ consists of infinitely many points. Let $x_{n}$ be the unique point of the one-point set $K \cap h^{\leftarrow}\left(y_{n}\right)$ for each $\mathrm{n}$. Then there exists a subsequence $\left\{x_{n_{i}}\right\}$ converging to some $x \in K$. Hence, $(t, x) \in Z$ and $\alpha(t, x)=t$.

(ii) $\alpha$ is a closed map. Assume that $F$ is closed in $Z$, and suppose that $t \in \operatorname{cl}(\alpha(F))$. Take a sequence $\left\{t_{n}\right\} \subset \alpha(F)$ converging to $t$. Then we can assume that $\left\{t_{n}\right\}$ consists of infinitely many distinct points, since otherwise $t=t_{n} \in \alpha(F)$ for some $n$. Hence, let

(2) $\left\{U_{n}\right\}$ be a disjoint open collection of $M$ such that $t_{n} \in U_{n}$ and $\operatorname{diam}\left(U_{n}\right)$ $<1 / 2^{n}$.

For each $n \in \omega$, take a point $z_{n} \in F$ such that $t_{n}=\alpha\left(z_{n}\right)$. Let $V_{n}$ be the $1 / 2^{n}$-neighborhood of $\beta\left(z_{n}\right)$ with respect to some metric of $X$. Take a point

(3) $w_{n}=\left(s_{n}, x_{n}\right) \in Z_{0} \cap\left(U_{n} \times V_{n}\right)$, since $z_{n} \in \operatorname{cl}\left(Z_{0}\right)$.

Then, by Lemma $1($ a) it holds that

(4) $s_{n}=f^{\leftarrow}\left(f\left(s_{n}\right)\right) \in U_{n}, x_{n}=h^{\leftarrow}\left(h\left(x_{n}\right)\right) \in V_{n}$, and $f\left(s_{n}\right)=h\left(x_{n}\right)$.

Note also that $\left\{s_{n}\right\}$ also converges to $t$, and hence let $K$ be a compact subset of $X$ such that (1) holds, where $y=f(t)$ and $y_{n}=f\left(s_{n}\right)=h\left(x_{n}\right)$.

By (2) and (4), it holds that $\left\{y_{n}\right\}$ consists of infinitely many distinct points. Let $x_{n}$ be the unique point of the one-point set $K \cap h^{\leftarrow}\left(y_{n}\right)$ for each $n \in \omega$. Then, there exists a subsequence $\left\{x_{n_{i}}\right\}$ converging to some $x \in K$. Hence, $(t, x) \in Z$ and $(t, x) \in F$, since $\left\{w_{n_{i}}=\left(s_{n_{i}}, x_{n_{i}}\right)\right\} \subset Z_{0}$ converges to $(t, x)$ and $\left\{z_{n_{i}}\right\}$ also converges to $(t, x)$ by (3) and (4). Hence, $t=\alpha(t, x) \in \alpha(F)$.

(iii) Each $\alpha^{\leftarrow}(t)$ is compact. We can assume that $t \notin M_{0}$, since $\alpha^{\leftarrow}(t)=$ $f^{\leftarrow}(f(t)) \times h^{\leftarrow}(f(t))$ is a one-point set when $t \in M_{0}$. By Fact 1 it suffices to show that $\operatorname{Int}\left(\alpha^{\leftarrow}(t)\right)=\varnothing$. Suppose that $U$ and $V$ are open sets of $M$ and $X$, respectively, such that

$$
K=(U \times V) \cap \alpha^{\leftarrow}(t) \neq \varnothing .
$$

Take a point $z \in K$. Then, $Z_{0} \cap(U \times V)=U \times V \cap\left(Z_{0} \backslash \alpha^{\leftarrow}(t)\right) \neq \varnothing$, since $z \in \operatorname{cl}\left(Z_{0}\right)$ and $z \in U \times V$. Therefore, $\operatorname{Int}\left(\alpha^{\leftarrow}(t)\right)=\varnothing$.

(iv) It can be shown by a parallel way that $\beta$ is a perfect onto map.

Corollary. Let $Y$ and $Z$ be a van Douwen-complete space and a Lašnev space, respectively. Suppose that $Y$ can be embedded as a subset of $Z$. Then, $Y$ is a $G_{\delta}$-subset of $Z$.

Proof. Assume that $Y \subset Z$ and let $q: T \rightarrow Z$ be a closed (irreducible) mapping from a metric space $T$ onto $Z$. Then, for the restricted closed mapping $q \mid q^{\leftarrow}(Y): q^{\leftarrow}(Y) \rightarrow Y$, let $h: X \rightarrow Y$ be an irreducible mapping given by Fact 
0 , where $X$ is a closed subset of $q^{\leftarrow}(Y)$. Hence, it holds that

(5) $X=q^{\leftarrow}(Y) \cap\left(\mathrm{cl}_{T} X\right)$.

By Theorem 1 it holds that $X$ is completely metrizable and hence the set $\mathrm{cl}_{T} X \backslash$ $X$ is an $F_{\sigma}$-subset in $T$. Put $\mathrm{cl}_{T} X \backslash X=\cup_{i} F_{i}$, where each $F_{i}$ is a closed subset in $T$. By (5) it holds that $F_{i} \subset T \backslash q^{\leftarrow}(Y)$ for each $i$. Since $q$ is a closed map, it holds that $q\left(\operatorname{cl}_{T} X\right)=\operatorname{cl}_{Z} Y$. Hence, $W=q\left(\operatorname{cl}_{T} X \backslash X\right)=\cup_{i} q\left(F_{i}\right)=\operatorname{cl}_{Z} Y \backslash Y$ is an $F_{\sigma}$-set in $Z$. Therefore, $Y=\left(\operatorname{cl}_{Z} Y\right) \cap(Z \backslash W)$ is a $G_{\delta}$-subset of $Z$, since $\operatorname{cl}_{Z} Y$ is a $G_{\delta}$-subset of $Z$.

\section{REMARKS AND EXAMPLES}

Remark 1. (a) There is much flexibility for the topologies of the original spaces, which yield the same quotient space by suitable decompositions (e.g., there are several ways to make a topological torus, using non-homeomorphic original spaces). Even using the same space, there are many different decompositions which yield the same decomposition space. For example, collapsing a single compact set $A$ in the Euclidian plane $E^{2}$, we have a quotient space $Y=$ $E^{2} / A$ which is homeomorphic to the original space $E^{2}$, when $A$ has the trivial shape (i.e., it has the shape type of a point). On the other hand, there are infinitely many compact nowhere dense subsets $A$ in $E^{2}$; all of them have the trivial shape, but are mutually non-homeomorphic. It can be said that our main theorem gives a property which every original space has even when the decomposition space is non-metrizable.

(b) Using the following decomposition theorem due to Lašnev [5], Van Doren [13] shows that the Baire category theorem is valid for every van Douwencomplete space.

Fact 4. Let $f: X \rightarrow Y$ be an irreducible closed onto map from a metric space $X$. Then, there exists a subspace $M$ of $Y$ such that $f \mid f^{\leftarrow}(M)$ is a perfect map and $Y \backslash M$ is $\sigma$-discrete in $Y$.

One of the remarkable points of this decomposition of the quotient space $Y$ is that the decomposition of Fact 4 does not depend on the maps $f$ and $h$ in Lemma 1 by Fact 1 . Namely, we have the following equality:

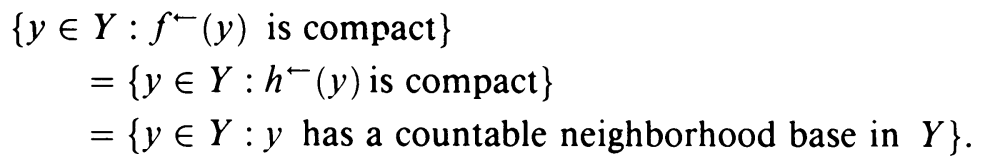

On the other hand, Stricklen Jr. [9] pointed out that the set $S_{0}=\{y \in Y$ : $f^{\leftarrow}(y)$ is a one-point set $\}$ is a dense $G_{\delta}$-subset of $Y$ so that the Baire category theorem for $Y$ also follows from this fact. Unfortunately, the second example $Y=E^{2} / A$ in (a) shows that $S_{0}$ is different from the set $H_{0}=\left\{y \in Y: h^{\leftarrow}(y)\right.$ is a one-point set $\}$ for an irreducible closed map $h$, in general. Lemma 1 , however, shows that their intersection $Y_{0}=S_{0} \cap H_{0}$ remains a large set (i.e., it is a dense $G_{\delta}$-subset of $Y$ ). In our proof of Theorem 1 the set $Y_{0}$ plays a crucial role.

(c) A topological property $P$ is said to be perfect provided it is both the invariant and inverse invariant of every perfect map. For example, completely metrizability and locally compactness are perfect topological properties. By the above proof of our Theorem 1 it holds that: Let $f: M \rightarrow Y$ be a closed irreducible onto map from a complete metric space $M$ which satisfies a perfect 
property $P$, and suppose that $h: X \rightarrow Y$ is an arbitrary closed irreducible map from a metric space $X$ onto $Y$. Then $X$ also satisfies $P$.

Remark 2. The van Douwen-complete spaces must have some harmonious properties. For example, there exists a universal space $D_{\alpha}$ for all van Douwencomplete spaces $X$ of $\operatorname{dim} X=0$ with the weight $w(M) \leq \alpha$ [11] (see also [12] for more general results). It is also known [10] that there are no universal spaces without assumption of complete metrizability of $M$. By virtue of our theorem we can show that $X$ can be embedded in $D_{\alpha}$ as a closed subset (see [12] for details).

Remark 3. By a method given in [7] we can show the following theorem.

Theorem 2. For any van Douwen-complete space $X$ there exists a perfect map $f$ from a strongly 0-dimensional van Douwen-complete space $Z$ onto $X$.

Proof. Oka [7] showed the following commutative diagram for every closed map $f$ from a metric space $M$ onto $X$, where $\operatorname{dim} Z=0, h$ is closed onto map, and both $p$ and $g$ are perfect onto maps.

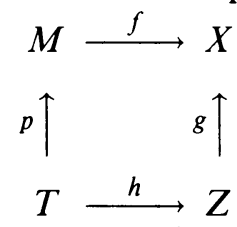

In our case the space $T$ is completely metrizable by Fact 3 , since $M$ is so and $p$ is perfect. Hence, $Z$ is a desired strongly 0-dimensional van Douwen-complete space.

The spaces $T$ and $Z$ used in the proof of Theorem 2 are constructed as the fiber products of some mappings (see [7] for details). The following example, however, shows that the fiber product $S=\{(t, x): f(t)=h(t)\}$ itself is too big for the proof of Theorem 1.

Example 1. If $Y$ is the quotient space obtained from the Euclidean plane $R^{2}$ by identifying the set of all the points on the $x$-axis to a point $y_{0}$, then the natural quotient map $\pi: R^{2} \rightarrow Y$ is closed irreducible (note also that $\pi^{\leftarrow}\left(y_{0}\right) \approx R$ ). Let $\alpha: S \rightarrow R^{2}$ be the natural projection map from the fiber product $S$ with respect to $f=h=\pi$. Then, $\alpha$ is not closed, since $\alpha^{\leftarrow}\left(\pi^{\leftarrow}\left(y_{0}\right)\right) \approx R^{2}$ and $\alpha \mid \alpha^{\leftarrow}\left(\pi^{\leftarrow}\left(y_{0}\right)\right)$ is not a closed map.

The following example shows that without the assumption of metrizability perfect pre-images of a van Douwen-complete space are neither van Douwencomplete nor Čech-complete, in general.

Example 2. Let $p: X \times C \rightarrow X$ be the natural projection map from the product space, where $X$ is the van Douwen-complete space in Example 1 and $C$ is a non-metrizable compact Hausdorff space. Then $p$ is obviously perfect and $X \times C$ is neither van Douwen-complete nor Čech-complete by Fact 3 and the following reason. Since every van Douwen-complete space has a $G_{\delta}$-diagonal, it is metrizable by Okuyama-Borges Theorem $[1,8]$ when it is assumed to be Cech-complete (note that in this case it is a paracompact $p$-space) .

Example 3. Let $X$ be the same van Douwen-complete space in Examples 1 and 2. Then, $X$ is not $G_{\delta}$ in its Stone-Čech compactification $\beta X$. Hence, our corollary does not holds for non-Lašnev space $Z$. 


\section{REFERENCES}

1. C. R. Borges, On stratifiable spaces, Pacific J. Math. 17 (1966), 1-16.

2. R. Engelking, Dimension theory, North-Holland, Amsterdam, 1978.

3. __ General topology, Heldermann Verlag, Berlin, 1989.

4. D. M. Hyman, A note on closed maps and metrizability, Proc. Amer. Math. Soc. 21 (1969), 109-112.

5. N. Lašnev, Continuous decompositions and closed mappings of metric spaces, Soviet Math. Dokl. 6 (1965), 1504-1506.

6. E. Michael, A note on closed maps and compact sets, Israel J. Math. 2 (1964), 173-176.

7. S. Oka, Every Lašnev space is the perfect image of a zero-dimesional one, Bull. Acad. Polon. Sci. Math. 28 (1980), 591-594.

8. A. Okuyama, Some generalizations of metric spaces, their metrization theorems and product spaces, Sci. Rep. Tokyo Kyôiku Daigaku Sect. A 9 (1967), 236-254.

9. S. A. Stricklen, Jr., An embedding theorem for Lašnev spaces, Gen. Topology Appl. 6 (1976), 145-152.

10. K. Tsuda, Non-existence of universal spaces for some stratifiable spaces, Topology Proc. 9 (1984), 165-171.

11. _ $A$ Aniversal space for strongly 0-dimensional closed images of complete metrizable spaces, unpublished manuscript.

12. __ Universal spaces for 0-dimensional van Douwen-complete spaces, preprint.

13. K. R. Van Doren, Closed, continuous images of complete metric spaces, Fund. Math. 80 (1970), 47-50.

Department of Mathematics, Ehime University, Matsuyama 790, Japan 